\title{
Risk Factors for Interstitial Cystitis in the General Population and in Individuals With Depression
}

\author{
M. Soledad Cepeda, Jenna Reps, Anthony G. Sena, Rachel Ochs-Ross \\ Janssen Research and Development, Titusville, NJ, USA
}

Purpose: To identify risk factors for interstitial cystitis (IC), a chronic bladder disorder that may have a significant detrimental impact on quality of life, in the general population and in individuals with depression.

Methods: This was a comparative study using a US claims database. Adults who had records of a visit to the health system in 2010 or later were included. The outcome was the development of IC within 2 years after the index date. The index date for the general population was the first outpatient visit, and for individuals with depression, it was the date of the diagnosis of depression. IC was defined using the concepts of ulcerative and IC. We included all medical conditions present any time prior to the index visit as potential risk factors.

Results: The incidence of IC was higher in individuals with depression than in the general population. Of the 3,973,000 subjects from the general population, 2,293 (0.06\%) developed IC within 2 years. Of the 249,200 individuals with depression, 320 $(0.13 \%)$ developed IC. The characteristics of the individuals who developed IC were similar in both populations. Those who developed IC were slightly older, more likely to be women, and had more chronic pain conditions, malaise, and inflammatory disorders than patients without IC. In the general population, subjects who developed IC were more likely to have mood disorders, anxiety, and hypothyroidism.

Conclusions: The incidence of IC was higher in individuals with depression. Subjects who developed IC had more chronic pain conditions, depression, malaise, and inflammatory disorders.

Keywords: Depression; Inflammation; Interstitial cystitis; Retrospective cohort study; Risk factors

- Research Ethics: The New England Institutional Review Board determined that studies conducted with the Optum database do not qualify as human subjects research (NEIRB \#12-286).

- Conflict of Interest: All authors are employees of Janssen Research \& Development, LLC. Janssen Research \& Development, LLC has an interest in depression. There is no mention of any medical product.

\section{INTRODUCTION}

Interstitial cystitis (IC) is a chronic bladder disorder characterized by pelvic pain, feelings of pressure, a persistent strong desire to void, increased urinary frequency, urinary urgency, and persistent symptoms despite antibiotic treatment [1]. It is a diagnosis of exclusion [2]. IC is often quite burdensome to the patient and may drastically affect quality of life [3].

IC is often described as a disease of the urothelium, and disruption of epithelial integrity and epithelial dysfunction have been proposed to explain its pathophysiology [4]. The aetiology of IC is not clear. Women are more likely to develop IC than men, and patients with IC have more chronic pain conditions and comorbidities than those without IC [5-7]. For example,
Corresponding author: M. Soledad Cepeda (iD https://orcid.org/0000-0002-5159-6217 Janssen Research and Development, 1125 Trenton Harbourton Rd, Titusville, NJ 08560, USA

E-mail: scepeda@its.jnj.com / Tel: +1-609-730-2413 / Fax: +1-609-730-7927

Submitted: July 27, 2018 / Accepted after revision: October 1, 2018
This is an Open Access article distributed under the terms of the Creative Commons Attribution Non-Commercial License (http://creativecommons.org/licenses/by-nc/4.0/) which permits unrestricted non-commercial use, distribution, and reproduction in any medium, provided the original work is properly cited. 
studies that assessed the co-occurrence of conditions with IC reported that patients with IC were more likely to have depression, chronic fatigue symptoms and panic attacks [8], but these parameters did not predict who would develop IC.

Since IC is a medical condition that is not well understood, using real-world evidence to identify risk factors in a large number of subjects before they develop IC would be helpful for understanding this condition. This study used a healthcare claims database to achieve this goal. Claims databases are created from billing and reimbursement transactions, and not for research purposes, but nonetheless can provide valuable information unavailable by other means [9].

We sought to assess the incidence of IC and to identify risk factors of IC.

\section{MATERIALS AND METHODS}

\section{Study Design}

We conducted a comparative descriptive study using retrospectively recorded data in a USA claims database, assessed the in- cidence of IC, and evaluated the risk factors of IC in a general adult population and an adult population with depression.

\section{Inclusion Criteria}

Subjects were included if they had records of a visit to the health system in 2010 or later and were 18 years or older. The first outpatient visit to the health system was the index date for the general population, and the diagnosis of depression was the index date for individuals with depression, respectively. We required that subjects had been continuously enrolled in the database for at least 1 year prior to the index date and for 2 years after (Fig. 1). We excluded subjects who had any codes for IC diagnosis or symptoms of cystitis 1 year before the index date.

\section{Medical Conditions}

All medical conditions assessed in this study were defined using Systematized Nomenclature of Medicine-Clinical Terms (SNOMED). SNOMED is a standardized, multilingual vocabulary of clinical terminology that is used by physicians and other health care providers for the electronic exchange of clinical

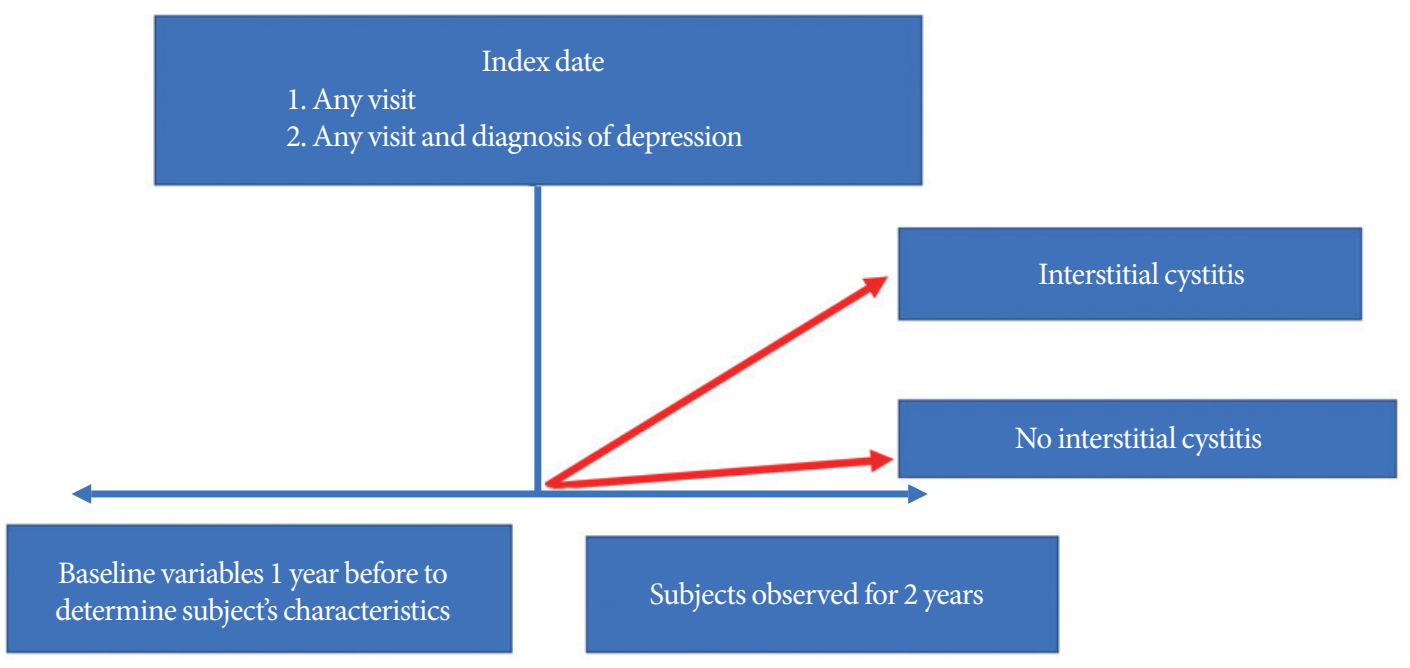

Fig. 1. Flow chart for assessing who did and did not develop interstitial cystitis and risk factors.

Table 1. SNOMED concepts used to define depression and the ICD-9 and ICD-10 codes used to define interstitial cystitis

\begin{tabular}{lc}
\hline SNOMED concepts for depression & ICD-9 and ICD-10 codes that mapped to SNOMED concepts for IC \\
\hline Adjustment disorder with depressed mood & N30.1 Interstitial cystitis (chronic) \\
Depressive disorder & N30.10 Interstitial cystitis (chronic) without hematuria \\
Dysthymia & N30.11 Interstitial cystitis (chronic) with hematuria \\
Adjustment disorder with depressed mood & 595.1 Chronic interstitial cystitis \\
\hline
\end{tabular}

SNOMED, Systematized Nomenclature of Medicine-Clinical Terms; ICD, International Classification of Diseases; IC, interstitial cystitis. 
health information [10].

IC was defined using the SNOMED concepts of ulcerative and IC. These SNOMED concepts contain the International Classification of Diseases (ICD)-9 and ICD-10 codes used in previous studies to define IC [11-13] which are listed in Table 1. The SNOMED concepts used to define depression are also listed in Table 1. These concepts do not include bipolar disorder.

\section{Data Source}

The Optum database (Optum Clinformatics Extended DataMart, Optum, Inc., Eden Prairie, MN, USA) is a USA adjudicated administrative health claims database for members with private health insurance, who are fully insured in commercial plans or have Medicare Advantage. It includes data captured from administrative claims processed from inpatient and outpatient medical services, as well as from dispensed prescriptions. Inpatient and outpatient medical claims are coded in the ICD-9-CM or ICD-10-CM.

\section{Outcome}

We assessed patients who developed IC within 2 years after the index date. We selected a 2-year window for 2 reasons. First, a longer window of risk could have introduced bias, since patients could leave the databases due to the IC diagnosis; in fact, the median time a patient was in the database was around 1.5 years. Second, a feasibility assessment showed that of all the IC cases present in the database after 2005, 67\% were captured within a 2 -year window.

\section{Risk Factors}

We included all medical conditions as potential risk factors. We assessed their presence at any time prior to the index date (including the index date). We calculated the prevalence of conditions in subjects who did and did not have a diagnosis of IC within 2 years after the index date and calculated the relative risk (RR) by dividing the prevalence of the condition in subjects with IC by the prevalence of the condition in subjects without IC. An RR higher than 1 means that the condition was more prevalent in subjects with IC. We also calculated 95\% confidence intervals (CIs).

The New England Institutional Review Board determined that studies conducted with the Optum database do not qualify as human subjects research (NEIRB \#12-286).

\section{RESULTS}

The incidence of IC was higher in individuals with depression than in the general population. Of the 3,973,695 subjects in the general population who met the inclusion criteria, 2,293 developed IC (0.06\%) within 2 years. Of the 248,822 individuals with depression who met the inclusion criteria, 320 developed IC $(0.13 \%)$ within 2 years.

Table 2. Prevalence and relative risk of risk factors of IC in the general population

\begin{tabular}{|c|c|c|c|c|c|}
\hline Variable & No. of subjects & IC (\%) & No IC (\%) & Relative risk & $95 \% \mathrm{CI}$ \\
\hline Age (yr) & $3,973,695$ & $50.87 \pm 16.86^{a)}$ & $47.47 \pm 18.30^{\mathrm{a})}$ & NA & NA \\
\hline Women & $2,011,471$ & 87 & 51 & 1.72 & $1.69-1.74$ \\
\hline Arthropathy & 936,258 & 37 & 24 & 1.55 & $1.47-1.63$ \\
\hline Inflammatory disorder of upper respiratory tract & 894,454 & 40 & 23 & 1.51 & $1.42-1.60$ \\
\hline Musculoskeletal pain & 644,634 & 27 & 16 & 1.65 & $1.54-1.76$ \\
\hline Malaise and fatigue & 374,332 & 19 & 9 & 1.99 & $1.83-2.17$ \\
\hline Mood disorder & 259,506 & 15 & 7 & 2.26 & $2.05-2.49$ \\
\hline Neuropathy & 299,105 & 15 & 8 & 1.96 & $1.77-2.16$ \\
\hline Anxiety & 212,877 & 12 & 5 & 2.27 & $2.03-2.53$ \\
\hline Headache disorder & 96,082 & 9 & 2 & 3.56 & $3.11-4.07$ \\
\hline Hypothyroidism & 287,478 & 13 & 7 & 1.83 & $1.64-2.03$ \\
\hline Low back pain & 266,694 & 13 & 7 & 1.87 & $1.68-2.09$ \\
\hline Lupus erythematosus & 7,759 & 1 & 0 & 4.71 & $3.07-7.21$ \\
\hline
\end{tabular}

IC, interstitial cystitis; CI, confidence interval; NA, not available.

${ }^{\text {a) }}$ Mean \pm standard deviation. 
Table 3. Prevalence and relative risk of risk factors of IC in individuals with depression

\begin{tabular}{|c|c|c|c|c|c|}
\hline Variable & No. of subjects & $\mathrm{IC}(\%)$ & No IC (\%) & Relative risk & $95 \% \mathrm{CI}$ \\
\hline Age (yr) & 248,822 & $49.95 \pm 15.10^{\mathrm{a})}$ & $48.99 \pm 17.59^{\mathrm{a})}$ & NA & NA \\
\hline Women & 168,467 & 94 & 68 & 1.39 & $1.36-1.43$ \\
\hline Arthropathy & 90,490 & 51 & 36 & 1.41 & $1.27-1.57$ \\
\hline Inflammatory disorder of digestive system & 51,362 & 33 & 21 & 1.61 & $1.37-1.88$ \\
\hline Inflammatory disorder of upper respiratory tract & 95,273 & 51 & 38 & 1.32 & $1.19-1.47$ \\
\hline Musculoskeletal pain & 67,062 & 38 & 27 & 1.43 & $1.24-1.64$ \\
\hline Headache disorder & 15,963 & 17 & 6 & 2.64 & $2.07-3.36$ \\
\hline Low back pain & 31,538 & 23 & 13 & 1.78 & $1.45-2.18$ \\
\hline Neuropathy & 35,731 & 23 & 14 & 1.57 & $1.28-1.92$ \\
\hline Malaise and fatigue & 52,464 & 29 & 21 & 1.38 & $1.16-1.64$ \\
\hline
\end{tabular}

IC, interstitial cystitis; CI, confidence interval; NA, not available.

${ }^{\text {a) }}$ Mean \pm standard deviation.

\section{Risk Factors}

The characteristics of the individuals who developed IC were similar in both the general population and in the population with depression (Tables 2, 3). Those who developed IC were slightly older, more likely to be women, and had more chronic pain conditions, such as arthropathy, musculoskeletal pain, low back pain, and neuropathic pain, than those who did not develop IC. A history of malaise and inflammatory disorders of the respiratory tract were also more prevalent in subjects with IC.

In the general population, subjects with IC were more likely to have diagnosis codes for mood disorders and anxiety. The prevalence of these conditions was more than twice as high in those who developed IC than in those who did not. Hypothyroidism and inflammatory disorders such as lupus were also seen more often in individuals who developed IC (Table 2).

Supplementary Tables 1 and 2 present lists of all the medical conditions that were statistically significantly more prevalent in subjects with IC in both populations.

\section{DISCUSSION}

We found that the incidence of IC was higher in individuals with depression and that the risk factors of IC were similar in individuals with and without depression. Those who went on to develop IC had more chronic pain conditions, malaise, and inflammatory disorders.

Data have been found in support of the proposal that inflammation plays an extensive role in depression, which has led to the concept of depression as an inflammatory condition [14-16]. We found that the incidence of IC was higher in individuals with depression. The findings of this study corroborate the theory that IC shares features of inflammatory autoimmune diseases [17].

We also found that chronic pain conditions, such as arthropathy, musculoskeletal pain, back pain, neuropathic pain, and headache disorders, were risk factors for IC. Functional and structural magnetic resonance imaging studies conducted in patients with IC, those with fibromyalgia, and healthy controls found that patients with IC and fibromyalgia shared a pattern of altered brain structure and function, with increased gray matter volume and functional connectivity in sensory, motor, and insular cortices [18]. Consequently, IC could be seen as a painful condition that persists due to pain centralization, a process where pain perception is decoupled from nociceptive inputs. Interventions targeting this centralization in the central nervous system could be beneficial [18].

A strength of this study is that the risk factors were assessed before the subjects developed IC, and therefore these variables are not confounded by the presence or the treatment of IC, which is the case in cross-sectional studies where risk factors and the disease of interest are measured at the same time. We found that symptoms of malaise/fatigue were more prevalent in individuals who developed IC and were not a consequence of having IC. Malaise and fatigue are cornerstone symptoms of chronic fatigue syndrome [19], which is often seen in patients with IC. Similarly, we found that inflammatory disorders of the digestive system were risk factors for IC. Irritable bowel syndrome is commonly reported in patients with IC [8].

We measured the incidence of IC; however, previous studies 
that have assessed the prevalence of IC have estimated a prevalence of $2 \%$ [1]. It is difficult to assess whether the estimate of incidence of IC in a 2-year time window is an underestimation or overestimation of IC. The clinical diagnosis of IC is challenging, because its symptoms are similar to those of other disorders of the bladder and there is no definite test to identify IC [20]. Furthermore, we do not know how healthcare providers utilized the codes we used in this study to define IC, and no validation studies have been conducted to assess how sensitive or specific the concepts used are. Errors in the classification of IC could lead to difficulties in identifying risk factors, which would affect the accuracy of the magnitude of the effect of the identified prognostic factors.

This study is based on an administrative claims database that contained data intended to facilitate the healthcare financial reimbursement process, not for research purposes. Therefore, not only is it possible that the accuracy of the diagnosis of medical conditions, as discussed above, could have been lower than in a prospective study, but also that the prevalence of the risk factors could have been less accurate. Another limitation is the inconsistent capture in claims databases of potential key variables that could be very predictive, such as biological markers or genetic data.

This study focused on the commercially insured population, which tends to be healthier than the general population. Although we expect that the risk factors found in this study would nonetheless generalize to other populations, we could have missed risk factors that are only observed in sicker individuals or among people who only have access to publicly available insurance.

In summary, individuals who went on to develop IC had more chronic pain conditions, depression, malaise, and inflammatory disorders than the general population.

\section{SUPPLEMENTARY MATERIALS}

Supplementary Tables 1 and 2 can be found via https://doi. org/10.5213/inj.1836182.091.

\section{AUTHOR CONTRIBUTION STATEMENT}

- Full access to all the data in the study and takes responsibility for the integrity of the data and the accuracy of the data analysis: $M S C, J R, A G S, R O R$

- Study concept and design: $M S C$, JR, ROR
- Analysis and interpretation of data: MSC, JR, AGS, ROR

- Drafting of the manuscript: $M S C$

- Critical revision of the manuscript for important intellectual content: JR, AGS, ROR

- Statistical analysis: $M S C, J R, A G S$

- Administrative, technical, or material support: AGS

- Study supervision: $M S C$

\section{REFERENCES}

1. Berry SH, Elliott MN, Suttorp M, Bogart LM, Stoto MA, Eggers P, et al. Prevalence of symptoms of bladder pain syndrome/interstitial cystitis among adult females in the United States. J Urol 2011;186: 540-4.

2. Homma Y, Ueda T, Tomoe H, Lin AT, Kuo HC, Lee MH, et al. Clinical guidelines for interstitial cystitis and hypersensitive bladder updated in 2015. Int J Urol 2016;23:542-9.

3. Dinis S, de Oliveira JT, Pinto R, Cruz F, Buffington CT, Dinis P. From bladder to systemic syndrome: concept and treatment evolution of interstitial cystitis. Int J Womens Health 2015;7:735-44.

4. Birder LA. Urinary bladder, cystitis and nerve/urothelial interactions. Auton Neurosci 2014;182:89-94.

5. Li GZ, Zhang N, Du P, Yang Y, Wu SL, Xiao YX, et al. Risk factors for interstitial cystitis/painful bladder syndrome in patients with lower urinary tract symptoms: a Chinese multi-center study. Chin Med J (Engl) 2010;123:2842-6.

6. Keller JJ, Chen YK, Lin HC. Comorbidities of bladder pain syndrome/interstitial cystitis: a population-based study. BJU Int 2012; 110(11 Pt C):E903-9.

7. Fan YH, Lin AT, Lu SH, Chuang YC, Chen KK. Non-bladder conditions in female Taiwanese patients with interstitial cystitis/hypersensitive bladder syndrome. Int J Urol 2014;21:805-9.

8. Clemens JQ, Elliott MN, Suttorp M, Berry SH. Temporal ordering of interstitial cystitis/bladder pain syndrome and non-bladder conditions. Urology 2012;80:1227-31.

9. Stang PE, Ryan PB, Racoosin JA, Overhage JM, Hartzema AG, Reich C, et al. Advancing the science for active surveillance: rationale and design for the Observational Medical Outcomes Partnership. Ann Intern Med 2010;153:600-6.

10. Reich C, Ryan PB, Stang PE, Rocca M. Evaluation of alternative standardized terminologies for medical conditions within a network of observational healthcare databases. J Biomed Inform 2012; 45:689-96.

11. Anger JT, Zabihi N, Clemens JQ, Payne CK, Saigal CS, Rodriguez LV. Treatment choice, duration, and cost in patients with interstitial 
cystitis and painful bladder syndrome. Int Urogynecol J 2011;22: 395-400.

12. Patel R, Calhoun EA, Meenan RT, O'Keeffe Rosetti MC, Kimes T, Clemens JQ. Incidence and clinical characteristics of interstitial cystitis in the community. Int Urogynecol J Pelvic Floor Dysfunct 2008;19:1093-6.

13. Wu EQ, Birnbaum H, Mareva M, Parece A, Huang Z, Mallett D, et al. Interstitial cystitis: cost, treatment and co-morbidities in an employed population. Pharmacoeconomics 2006;24:55-65.

14. Cepeda MS, Stang P, Makadia R. Depression is associated with high levels of C-reactive protein and low levels of fractional exhaled nitric oxide: results from the 2007-2012 National Health and Nutrition Examination Surveys. J Clin Psychiatry 2016;77:1666-71.

15. Berk M, Williams LJ, Jacka FN, O’Neil A, Pasco JA, Moylan S, et al. So depression is an inflammatory disease, but where does the inflammation come from? BMC Med 2013;11:200.

16. Miller AH, Raison CL. The role of inflammation in depression: from evolutionary imperative to modern treatment target. Nat Rev Immunol 2016;16:22-34.

17. Logadottir Y, Delbro D, Lindholm C, Fall M, Peeker R. Inflammation characteristics in bladder pain syndrome ESSIC type 3C/classic interstitial cystitis. Int J Urol 2014;21 Suppl 1:75-8.

18. Kutch JJ, Ichesco E, Hampson JP, Labus JS, Farmer MA, Martucci KT, et al. Brain signature and functional impact of centralized pain: a multidisciplinary approach to the study of chronic pelvic pain (MAPP) network study. Pain 2017;158:1979-91.

19. Avellaneda Fernández A, Pérez Martín A, Izquierdo Martínez M, Arruti Bustillo M, Barbado Hernández FJ, de la Cruz Labrado J, et al. Chronic fatigue syndrome: aetiology, diagnosis and treatment. BMC Psychiatry 2009;9 Suppl 1:S1.

20. You S, Yang W, Anger JT, Freeman MR, Kim J. 'Omics' approaches to understanding interstitial cystitis/painful bladder syndrome/ bladder pain syndrome. Int Neurourol J 2012;16:159-68. 